\title{
Prototipagem de Serviço relacionada à Gamificação e Gestão de Design por uma Abordagem Sistêmica: análise bibliométrica
}

\author{
Prototyping service related to gamification, Design management and systemic \\ approach: bibliometric analysis
}

FIGUEIREDO, Luiz Fernando Gonçalves de; Pós-Doutor; UFSC - NAS Design

Iffigueiredo2009@gmail.com

FERREIRA, Alais Souza; Mestranda em Design; UFSC - NAS Design

alais.ferreira@live.com

CONTI, Paolo; Mestrando; Universidade Federal de Santa Catarina - Animaking

paolo@animaking.com.br

\section{Resumo}

O Design de Serviço (DS) disponibiliza métodos centrados no usuário que podem contribuir para a inovação e prototipagem de serviços (PS), que permite visualizar os processos e interações entre os atores. A gamificação pode favorecer a PS ao beneficiar a comunicação entre esses atores, e a Gestão de Design (GD) por viabilizar uma nova forma de experienciar o já vivenciado. Devido a GD possuir visão holística pode-se deduzir que esta possui Abordagem Sistêmica (AS). Assim, este artigo objetiva revisar as pesquisas realizadas sobre o processo de PS por meio da gamificação, com vistas à GD por uma AS. A pesquisa caracteriza-se como teórica, quantitativa e descritiva, constituída por Revisão Sistemática da Literatura (RSL). Como resultado identificaram-se três artigos, que relacionam todas as áreas mencionadas, pertencentes aos autores Patrício (2016), Klapztein e Cipolla (2016) e Rau, Zbiek e Jonas (2017). Estas pesquisas utilizaram a gamificação como ferramenta ou método gamificado para a PS.

Palavras Chave: prototipagem de serviço; gamificação; gestão de design; abordagem sistêmica.

\begin{abstract}
The service Design (SD) provides user-centric methods that can contribute to the innovation and prototyping of services (PS), which allows you to visualize the processes and interactions among the actors. Gamification can favor PS by benefiting communication between these actors, and Design Management (DM) for enabling a new way to experience the already experienced. Because DM has a holistic view it can be deduced that it has a systemic approach (AS). Thus, this article aims to revise the research carried out on the PS process through gamification, with a view to DM for an AS. The research is characterized as theoretical, quantitative and descriptive, consisting of a systematic revision of the literature (RSL). As a result, three articles were identified, which relate all the areas mentioned, belonging to the authors patrician (2016), Klapztein and Cipolla (2016) and Rau, Zbiek and Jonas (2017). These surveys used gamification as a tool or Gamificado method for PS.
\end{abstract}

Keywords: service prototyping; gamification; design management; systemic approach. 


\section{Introdução}

O DS pode ser considerado holístico, co-criativo, sequencial, evidenciado e multidisciplinar, por integrar as áreas de marketing, design, gestão de empresa e pesquisa de cliente. Esta abordagem nos ajuda a inovar, criar novos ou aperfeiçoar serviços, tornando-os mais úteis, usáveis e desejáveis para os clientes, eficiente e eficaz para as organizações. (MORITZ, 2005; STICKDORN; SCHENEIDER, 2011). Os métodos de DS podem ser centrados no usuário e contribuir para a inovação e prototipação de serviços que levam em consideração as necessidades e desejos das pessoas, bem como as restrições e possibilidades dos provedores de serviços (BOHREN et al.; 2015). A prototipagem dá forma a uma ideia, nos permitindo aprender e aperfeiçoá-la. Segundo Blomkvist (2011) a palavra protótipo deriva do grego prototypos, que é a junção dos termos proto "primeiro" e typos "impressão", e pode ser utilizada em diferentes contextos e disciplinas como no design. Os protótipos de serviço permitem visualizar os processos e interações ao invés de objetos tangíveis, possibilitando o mapeamento e a compreensão de todo o processo para os atores envolvidos.

A gamificação pode favorecer a PS ao beneficiar a comunicação entre esses atores e a visualização do processo para estes. O termo gamificação foi criado entre 2002 e 2003 por Nick Pelling, ganhando popularidade oito anos depois quando McGonigal (2012) realizou uma apresentação de TED. No início do século XX, devido ao surgimento da gamificação, o jogo deixou de ser considerado uma atividade destinada à distração e passou a ser utilizado pelos negócios como uma forma de adquirir conhecimento, desenvolvimento pessoal e profissional. Devido à influência da gamificação na mudança do comportamento humano, esta pode ser utilizada na GD para privilegiar a troca de conhecimentos e informações entre os diversos setores das empresas. Assim, o principal objetivo da gamificação, inserida no processo de GD como ferramenta, é viabilizar uma nova forma de experiência ao praticar o já vivenciado, induzir o trabalhador ou usuário a ver de maneira diferenciada e divertida as tarefas que deve realizar. A AS pode ser incluída nesse contexto para ampliar a visão da PS, gamificação e da GD, podendo auxiliar estas a visualizarem o todo.

Assim, questiona-se: A gamificação vem sendo utilizada como ferramenta ou método para prototipar serviços? Por isso, objetiva-se revisar, junto à literatura qualificada, pesquisas realizadas sobre o processo de PS por meio da gamificação, com vistas à GD por uma AS. Para isso, foi necessário: Identificar os periódicos que vêm publicando resultados de pesquisa que relacionam as áreas mencionadas anteriormente; Especificar as abordagens das publicações encontradas; e Detectar os principais grupos de pesquisa, pesquisadores, instituições e países. A pesquisa é caracterizada como básica, teórica, quantitativa, descritiva e como levantamento bibliográfico de dados secundários por tratar-se de uma RSL de caráter exploratório. Para Hoss (2014, p. 21), "embora o potencial de criação de protótipos de serviço seja explícito para a área, a prática real ainda precisa ser melhor estudada". Logo, o autor informa sobre a existência de lacunas de pesquisas sobre o processo de PS não só no Brasil, como também internacionalmente. Isto justifica o desenvolvimento da pesquisa, bem como a relevância e contribuição deste artigo para a área.

\section{Design de Serviço (DS): prototipagem}

O DS é uma abordagem que nos ajuda a inovar, criar novos, ou aperfeiçoar serviços, tornando-os mais úteis, usáveis e desejáveis para os clientes, e eficiente bem como eficaz para as organizações. Pode ser considerado um campo holístico, multidisciplinar e integrativo, por integrar as áreas de marketing, design, gestão de empresa e pesquisa de cliente (MORITZ, 2005). Segundo Bohren et al. (2015, p.5), o DS pode nos beneficiar na aplicação de "métodos centrados no usuário para inovar e dar forma aos serviços 
organizados em torno das necessidades e desejos das pessoas, levando em consideração as restrições e as possibilidades dos provedores de serviços e ambientes". Stickdorn e Scheneider (2011) complementam ao destacarem cinco princípios para o DS: centrado no usuário, co-criativo, sequencial, evidenciado e holístico. De acordo com Rau, Zbiek e Jonas (2017, p. 49), o processo de DS: "se concentra no usuário; promove uma visão sem restrições do problema; reconhece a necessidade de uma perspectiva colaborativa e sistêmica; e enfatiza a importância das visualizações no processo de design".

Para Kotler (2005), serviço é qualquer ato ou desempenho intangível que uma parte possa oferecer a outra, sem resultar na propriedade de nada, e que pode ou não estar ligado a produtos físicos. Devido a isto, é complicado testar e avaliar as características de um serviço, já que o mesmo não possui uma forma física, principalmente serviços baseados em complexas interações sociais. Por isso, "as ideias complexas [...] requerem protótipos que possam ser liberados em campo para ver como sobrevivem e se adaptam", que seria a Prototipagem de Serviço (PS) (BROWN, 2010, p.93). Acredita-se que a prototipagem beneficia o processo de design e a produção. Rau, Zbiek e Jonas (2017, p. 54) mencionam que os protótipos de serviço: permitem visualizar os processos e as interações ao invés de objetos tangíveis; são "extremamente úteis para a ideação" colaborativa; e "podem ajudar a superar os desafios apresentados pela imaterialidade e a interatividade dos serviços", permitindo aos designers mapearem o processo completo.

Zimmer Santos e Hoffmann (2016) informam que por meio do protótipo é possível estabelecer o escopo do serviço a ser implantado, para o qual todo o projeto será elaborado. Quando relacionado com o serviço, produzir um protótipo torna-se "uma maneira informativa para designers explorarem quais seriam as experiências para uma determinada pessoa, num contexto específico" (CASTRO, 2014, p.77). O protótipo proporciona "uma verdadeira experiência para os usuários [...] que exige um grau de resolução e funcionalidade condizente com o contexto cotidiano, e plenamente integrado à vida das pessoas" (CASTRO, 2014, p. 79). Por isso, denomina-se como prototipagem da experiência que é "aplicada para demonstrar o contexto, identificar problemas e oportunidades de design. [...] o objetivo da prototipagem é alcançar uma simulação de alta fidelidade de uma experiência existente, que não pode ser experimentada diretamente, porque é insegura, indisponível, custa caro" (CASTRO, 2014, p. 76). Hoss (2014) compila em um infográfico as taxonomias de protótipos (Figura 1).

Figura 1 - Taxonomia de protótipos

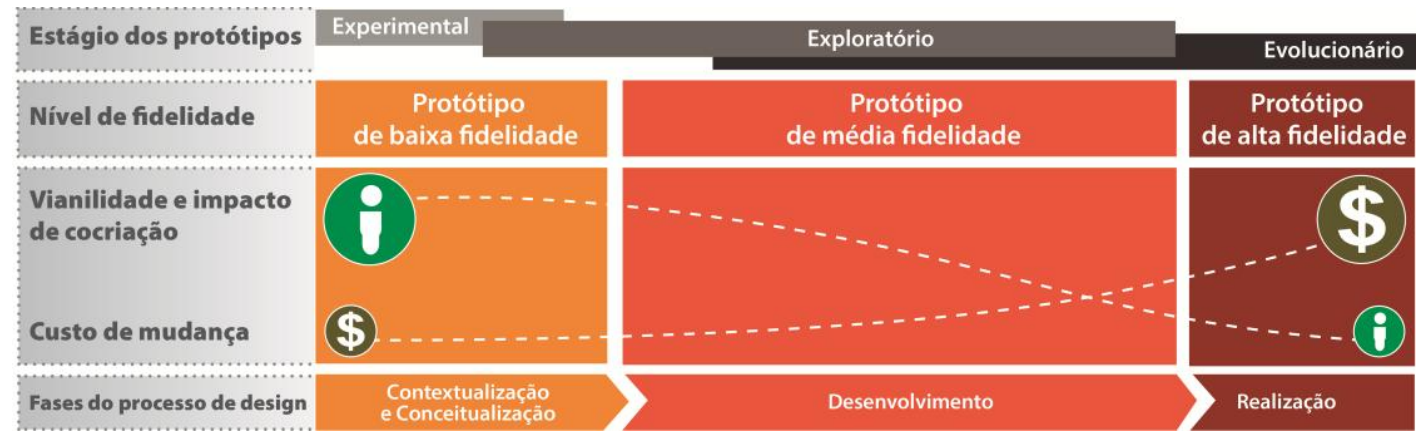

Fonte: Hoss (2014) com base em Buddeet al., (1992); Aguiar et al (2008); Alcoforado (2008)

A prototipagem, relacionada aos serviços, busca simular as experiências considerando "o provedor do serviço, artefatos envolvidos, o usuário e suas expectativas", e o ambiente (HOSS, 2014, p. 22). Assim, os protótipos tornam visíveis as ideias de serviços para os atores envolvidos, já que "ter alguma representação tangível fornece um ponto de referência comum que permite que os interessados em colaborar e avaliar forneçam sugestões para aperfeiçoar o projeto" de serviços (HOSS, 2014, p. 45). A gamificação pode auxiliar na comunicação entre estes atores, por isso a próxima seção aborda esta área. 


\section{Gamificação}

O conceito de gamificação tem origem na área de Design de Jogo (DJ) que, segundo Salen e Zimmerman (2004), é uma atividade que cria e define estruturas e regras de jogo, visando à geração de experiências significativas. Os autores, juntamente com Klapztein e Cipolla (2016), informam que os designers de jogos têm desenvolvido estratégias para promover o engajamento, o prazer e uma variedade de sensações nas experiências de jogos. Estes têm aplicado suas pesquisas em áreas além dos jogos, como naquelas relacionadas ao serviço (em que os designers buscam melhorar a experiência do usuário e o envolvimento em serviços).

Alguns pesquisadores já veem relacionando os campos do DJ com o de serviços. Huotari e Hamari (2012, 2016) apresentam a gamificação a partir da perspectiva do marketing de serviços como um processo de melhoria do serviço. Essa fusão pode fornecer uma estrutura sobre como a jogabilidade pode fazer parte ou dar suporte aos serviços, ou ainda ajudar no desenvolvimento de serviços gamificados. Os autores relatam que os jogos podem ser considerados sistemas que exigem o envolvimento ativo dos usuários em que a qualidade do serviço é determinada pela qualidade funcional da experiência. Para Klapztein e Cipolla (2016) os elementos de DJ podem ser descritos como serviços e os jogos como sistemas de serviço. Os autores reuniram alguns termos relacionados ao DJ, e seus respectivos autores, que visam aplicar os conceitos de DJ com base nas práticas de desenvolvimento de jogos, fora do contexto de jogos para aumentar o engajamento, entre esses termos estão o: "Gamestorming (Gray \& Macanufo, 2010), Game-Based Marketing (Zichermann \& Linder, 2010) e [...], Gamification (Deterding, Khaled, et al., 2011; Huotari \& Hamari, 2012; Law, Kasirun, \& Gan, 2011; Michaelides, 2011; Simões, Redondo \& Vilas, 2013; Teresa \& Kramer, 2011; Thom, Millen, \& DiMicco, 2012)" (KLAPZTEIN; CIPOLLA, 2016, p. 569).

A gamificação pode ser compreendida como a aplicação de elementos, mecanismos, dinâmicas e técnicas de jogos no contexto fora do jogo com o "objetivo de resolver problemas práticos ou de despertar engajamento entre um público específico", não sendo necessariamente desenvolver um jogo (COHEN, 2011; VIANNA et al., 2013, p.13; NAVARRO, 2013). Os elementos, mecânicas, dinâmicas e técnicas incluem o lançamento de desafios, cumprimento de regras, metas claras e bem definidas, efeito surpresa, linearidade dos acontecimentos, conquista por pontos e troféus, estatísticas e gráficos com o acompanhamento da performance, superação de níveis e criação de avatares. Já o contexto fora do jogo remete a situações pertencentes à realidade do dia a dia profissional, escolar e social do indivíduo. Para Olenski (2014) essa abordagem está em crescente aceitação como um método de resolução de problemas e vêm sendo utilizada para melhorar o envolvimento dos usuários.

Conforme Patrício (2016, p. 2), "um método e uma ferramenta gamificados podem influenciar o 'bom' comportamento dos funcionários no local de trabalho" por: desencadear a curiosidade das pessoas pela inovação; manter as pessoas intrinsecamente motivadas para se envolver continuamente; aprimorar a colaboração interna; promover maior diversão, compromisso, abertura a novas ideias e tecnologias entre os funcionários; aumentar a motivação para aprender, crescer, e arriscar; fornecer ideias para futuras áreas de aplicações de produtos ou serviços; educar sobre como aceitar o fracasso; entre outros comportamentos.

Assim, a gamificação pode ser uma ferramenta a disposição do design para simplificar um trabalho ou melhorar o processo de um serviço. Segundo Dallagnol (2016), existe a possibilidade desta ferramenta ser utilizada no processo de GD para atingir um novo método de atender o usuário, de forma que ele aproveite o conteúdo disponibilizado por meio de estratégias utilizadas pelo jogo, buscando motivá-lo e até influenciá-lo psicologicamente em suas decisões. $\mathrm{O}$ autor propõe que a GD utilize a gamificação para suprir as necessidades, com a troca de conhecimentos e informações, tanto de empregado para empregado, quanto de empregado para administrador, captando melhorias que podem ser implementadas na empresa. Então, o objetivo da gamificação, inserida no processo de GD, é viabilizar uma nova forma de experiência ao praticar o já vivenciado e induzir o trabalhador ou usuário a ver de maneira diferenciada e divertida as tarefas que deve realizar. 


\section{Gestão de Design (GD)}

$\mathrm{Na}$ contemporaneidade o reconhecimento da ocorrência de diversidades geográficas, socioculturais e econômicas no Brasil, são fatores que têm evidenciado o Design, posicionando-o como responsável pelo desenvolvimento de projetos de produtos, serviços, ambientes e experiências, considerando toda a complexidade e aspectos peculiares às organizações comerciais. Segundo Aros e Figueiredo (2015), o design envolve pesquisas, processos, práticas centradas nas pessoas, e a gestão é um processo sistemático, que define, planeja, desenvolve, implementa e controla ações de design. Assim, para Mozota (2011, p. 95), o conceito da GD está relacionado à implementação do "design como um programa formal de atividades dentro de uma corporação por meio da comunicação da relevância do design para as metas corporativas de longo prazo e da coordenação dos recursos de design em todos os níveis de atividade corporativa para atingir os" objetivos. Por isso, a GD pode coordenar recursos de design nos níveis operacionais (projetos), organizacionais (departamentos) e estratégicos (missão). Isso pode contribuir para a mudança do comportamento e da visão corporativa. A GD possui os objetivos: "1. Treinar parceiros ou gerentes e designers. Isso implica familiarizar os gerentes com o design e os designers com a gestão. 2. Desenvolver métodos de integração do design no ambiente corporativo." (MOZOTA, 2011, p. 92). Desta forma, o gerenciamento por meio de estratégias planejadas, métodos, técnicas e ferramentas de Design podem oferecer vantagens competitivas à empresa.

Logo, deduz-se que a GD possui abordagem holística e sistêmica ao integrar produção de bens, serviços, marketing, recursos humanos, meio ambiente, qualidade de vida, ética e responsabilidade social, com visão técnica e estratégica. Com isso em vista, Martins \& Merino (2011) relatam a importância da integração da GD com outras áreas em todas as fases do processo de desenvolvimento e produção, incluindo a concepção de estratégias para uma empresa, sua realidade local, vocação e objetivos. Para Mozota (2011), a GD possui duas abordagens a administrativa e a estratégica. A abordagem administrativa requer a aplicação das diferentes teorias de gestão científica, comportamental, situacional, de decisão e sistemática, conceitual e prática para enriquecer os métodos de GD. Já a abordagem estratégica "envolve a compreensão do design como um novo paradigma a fim de chegar a ideias e métodos que possam ser usados para aumentar a eficiência da gestão como um todo" (MOZOTA, 2011, p.100). Além disso, o autor afirma que o design busca por ferramentas específicas para formulação estratégica, gestão de ideias, modelos e protótipos para inovação, bem como ferramentas de comunicação para ampliar fronteiras. Assim sendo, a GD controla a administração do valor agregado do design, dos relacionamentos e dos processos, auxiliando na identificação dessas ferramentas que pode ser a gamificação ou os métodos gamificados. Pelo fato dessa disciplina possuir visão holística, a próxima seção relata o conceito da abordagem sistêmica.

\section{Abordagem Sistêmica (AS)}

A AS transfere o foco dos objetos para o estudo global dos sistemas envolvendo todos os processos e interdependências, pois cada um dos elementos ao serem reunidos para construir uma unidade funcional maior, desenvolvem qualidades que não se encontram nos componentes isolados (BERTALANFFY, 2014). Essa visão é considerada holística por conceber o mundo como um todo integrado. Segundo Capra (2010), a ênfase nas partes é chamada de mecanicista ou reducionista; a ênfase no todo, de holística, organísmica ou sistêmica.

A AS pode ampliar a visão de GD, entendendo a organização "como um sistema aberto, em que as interferências ou problemas detectados podem ser de ordem ambiental, social ou econômica, relativos a interferências e relações internas e externas à organização e ao sistema" de GD (SILVA; FIGUEIREDO, 2010, p. 9). Assim, segundo Silva e Figueiredo (2012), as teorias de design, de GD e de AS de Design, quando aliadas, ajudam a entender como é ter uma perspectiva sistêmica do contexto (SILVA; FIGUEIREDO, 2012). 
A AS inserida no design mostra como deve acontecer o trabalho dentro do processo de criação do serviço, aperfeiçoando-o e entendendo que cada parte possui sua importância e deve estar presente dentro de um sistema maior. Nessa interação dinâmica das partes é necessário compreender os processos isoladamente, bem como os problemas encontrados na organização e na ordem que os unifica.

\section{Procedimentos Metodológicos}

O artigo é caracterizado por sua natureza como pesquisa básica e teórica; pela abordagem como quantitativo; pelos objetivos como descritivo; pelos procedimentos metodológicos como levantamento bibliográfico de dados secundários (GIL, 2010). Trata-se de uma Revisão Sistemática da Literatura (RSL) de caráter exploratório que visa levantar as pesquisas científicas realizadas no processo de PS por meio da gamificação, com vistas à GD por uma AS. A Figura 2 apresenta o processo executado na RSL.

Figura 2 - Diagrama de bloco da RSL

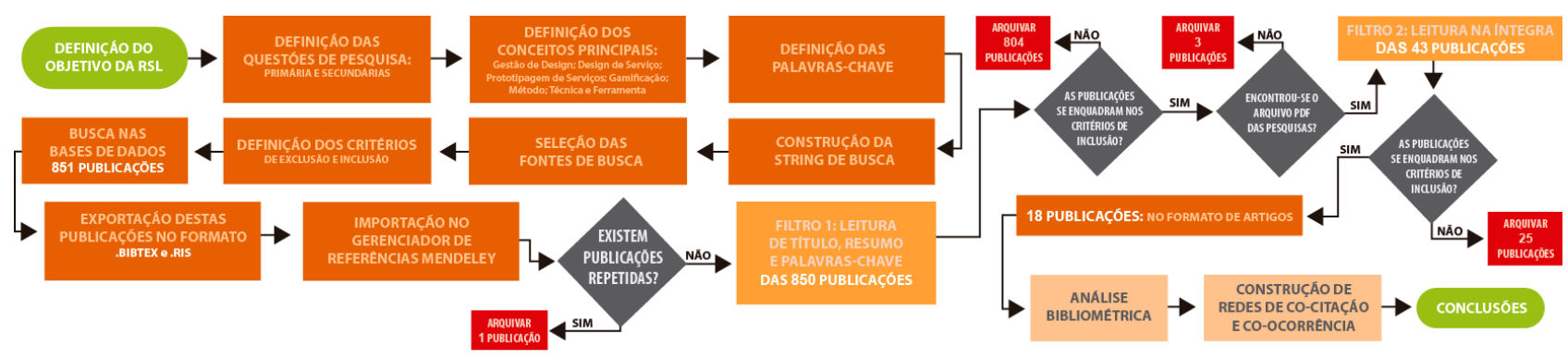

Fonte: Elaborado pelos autores com base no processo de Conforto, Amaral e Silva (2011)

O processo começou com a construção do protocolo de pesquisa para realizar a busca nas bases de periódicos Scopus, Scielo, Web of Science e ProQuest (selecionadas pela relevância para a área pesquisada), no período de 24 de outubro a 14 de novembro de 2017, com a string apresentada na Figura 3. Foram buscados artigos, capítulos de livrose livros publicados de 2012 a 2017, e que estavam dispostos nas áreas de ciências sociais ou ciências sociais aplicadas.

Figura 3 - Resultado das buscas

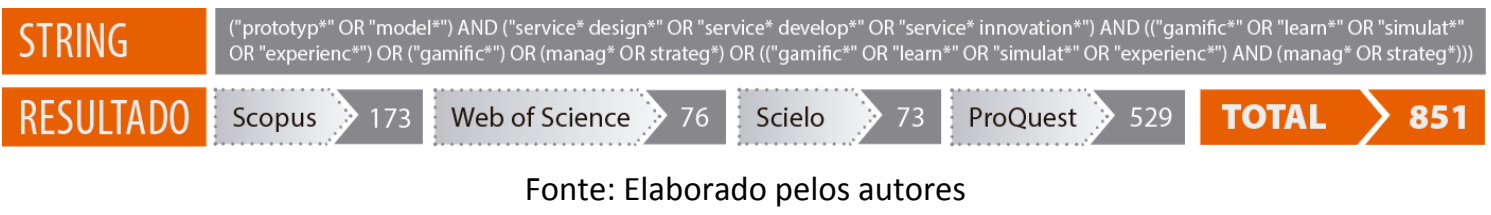

Após, foi estabelecido os critérios de inclusão quanto ao: ESCOPO (processo de PS por meio da gamificação ou outro meio, com vistas à GD ou por uma AS); TPO DE REFERÊNCIA (artigos de periódicos, anais de eventos, livros e capítulos de livro); ACESSO (por meio do Portal de Periódicos da CAPES na instituição UFSC; Google Acadêmico; ou Portal das editoras, de forma gratuita); e IDIOMA (Inglês e Português). Para o processo de seleção, as pesquisas foram importadas no gerenciador de referências Mendeley, que permitiu verificar a existência de referências duplicadas. Foram realizadas duas filtragens sequenciais (descritas na Figura 2) e os resultados obtidos em cada filtragem estão dispostos na Figura 4.

Figura 4 - Resultado das buscas de Artigos, Livros e Capítulos de Livros
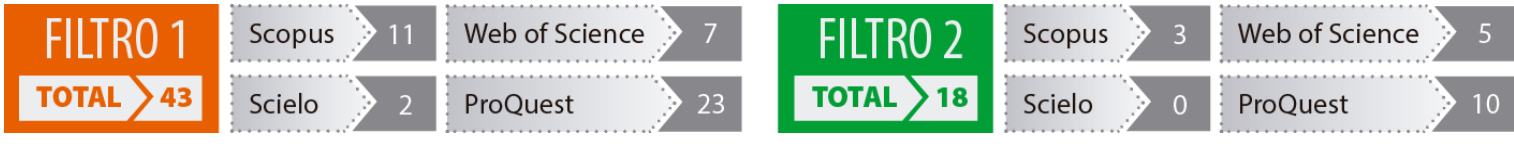

Fonte: Elaborado pelos autores 
Por fim, obteve-se o portfólio final de referências composto apenas por Artigos, apresentado na próxima seção, pois não foi possível encontrar Livros e Capítulos de Livros relevantes.

\section{Resultados}

Este item aborda gráficos que sintetizam o portfólio final das referências (Figuras 5 e 6) e uma subseção referente à análise dos resultados quantitativos.

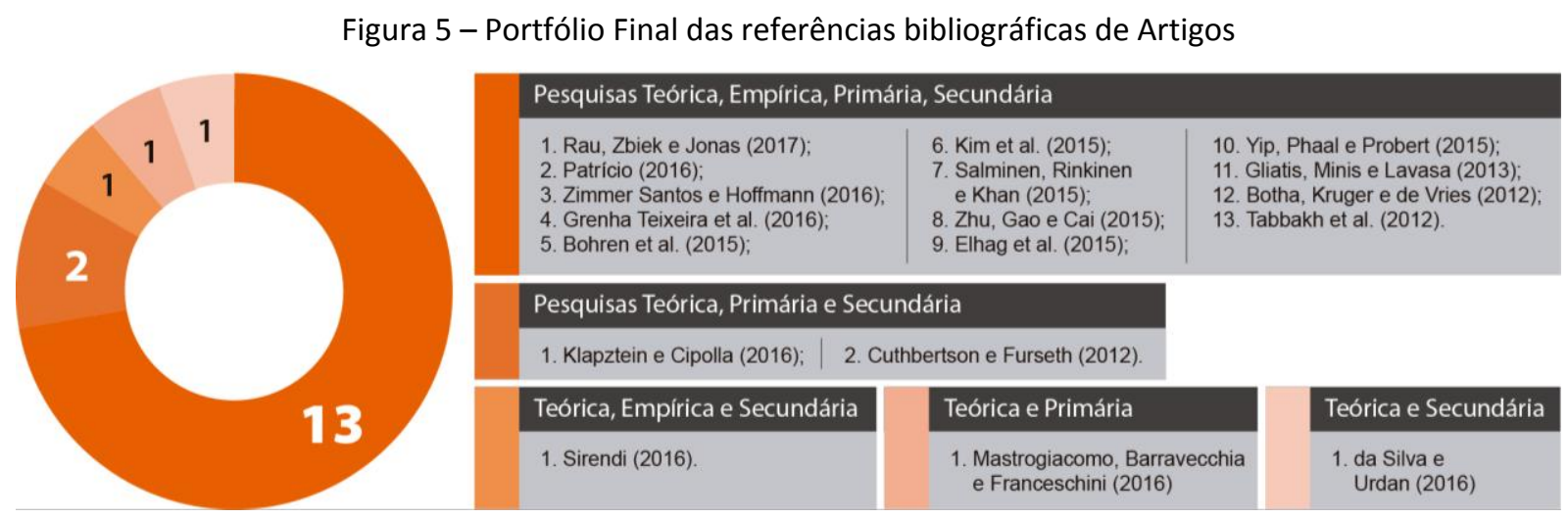

Fonte: Elaborado pelos autores

Figura 6 - Pesquisas de Prototipagem de Serviço (PS) relacionadas com a Gamificação, GD e AS.
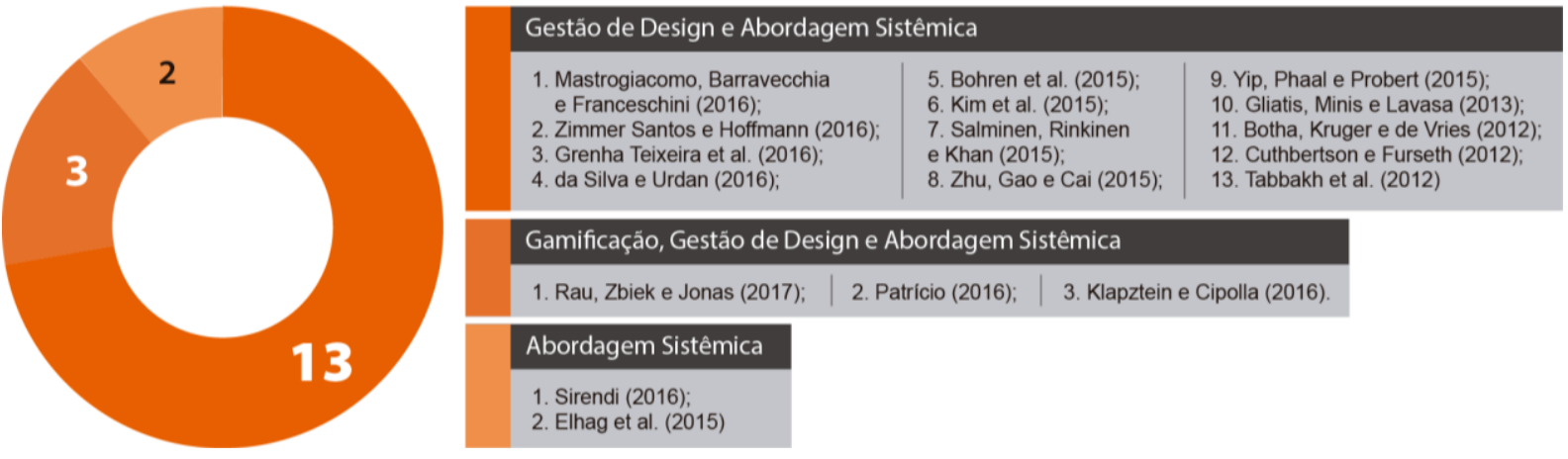

Fonte: Elaborado pelos autores

A Figura 6 evidencia que apenas três artigos relacionam a PS com todas as áreas estudadas (Gamificação, GD e AS), por isso, apresenta-se na sequência um resumo somente destas pesquisas. A primeira pesquisa, do pesquisador Patrício (2016), aborda o método gamificado IdeaChef (jogo de tabuleiro que usa metáforas de culinária) que integra conceitos de design de serviços com técnicas de resolução de problemas e inovação empresarial, para converter ideias de alto potencial em conceitos de trabalho ou protótipos.

A segunda pesquisa, dos pesquisadores Klapztein e Cipolla (2016), remete ao modelo de aplicação do Gamification Service Framework (GSF - Estrutura de Gamificação do Serviço) que pode ser entendido como um método de prototipagem rápido que combina a metodologia de ADR (Pesquisa-Ação em Design), Design de Jogo (Gamification) e Design de Serviço (Jornada do Serviço) para gerenciar serviços de forma simples.

E a terceira pesquisa, dos pesquisadores Rau, Zbiek e Jonas (2017), relata sobre o protótipo de serviço chamado como "role-playing" (jogo de interpretação de papeis ou jogo de 
representação) que é usado para simular interações entre clientes e funcionários em que os planos de serviços fornecem gráficos de processo das atividades de entrada e saída necessárias para entregar o serviço. A próxima subseção aborda a articulação da análise bibliométrica com a análise de redes de co-citação (de fontes e autores) e co-ocorrência de termos.

\subsection{Análise Bibliométrica e Redes}

Esta subseção aborda a análise bibliométrica (Figura 7 e Figuras 9 à 11) e a construção de redes (Figuras 8, 9 e 11) a partir do Portfólio Final de 18 referências. Destas referências, 13 foram encontradas na base Scopus e uma lista foi criada no próprio site. Além da análise bibliométrica disponibilizada na Scopus, foi desenvolvido um conjunto de redes com o auxílio do software VosViewer (2017) a partir da lista criada, esta foi exportada em um arquivo ".csv" com todos os dados de cada referência. Este arquivo foi aberto no VOSviewer para a criação das redes de co-citação (Figuras 8 e 9) e co-ocorrência (Figuras 11 à 15). A Figura 4 mostra as principais fontes do portfólio.

Figura 7 - Principais fontes do portfólio e a Evolução dos respectivos FI - SJR

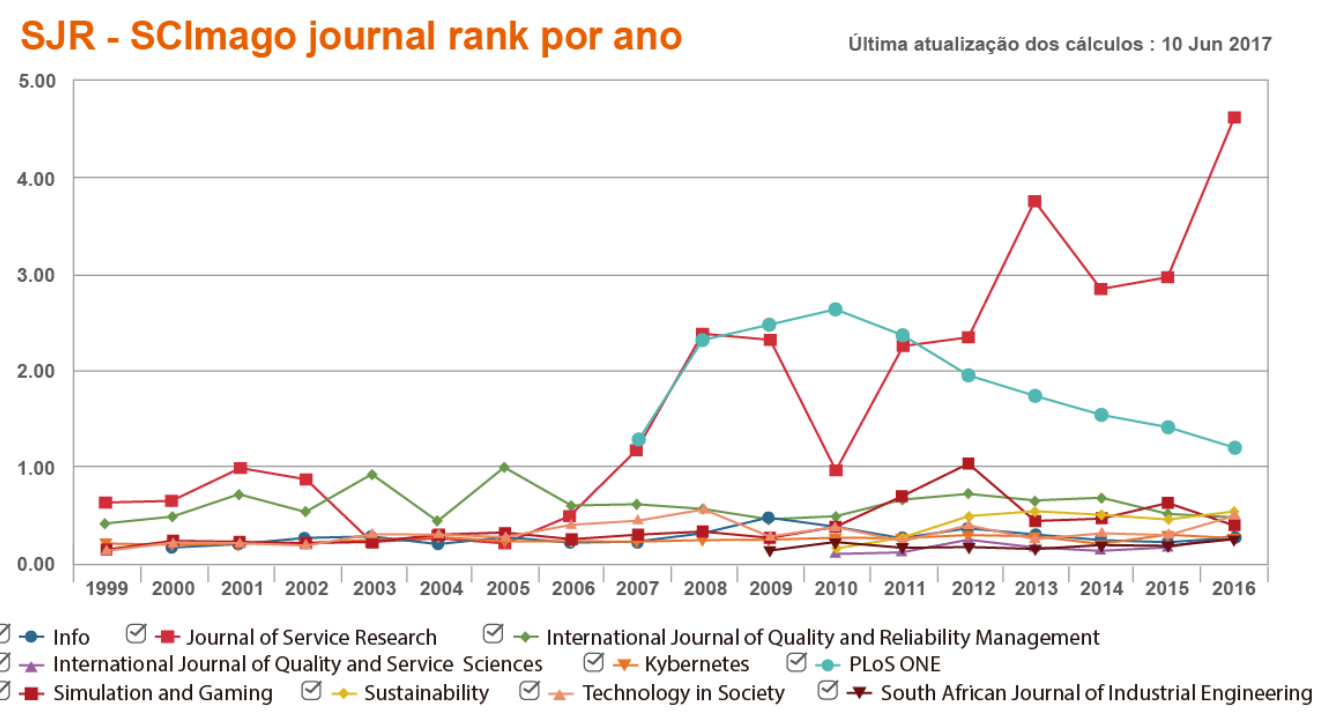

Fonte: Scopus (2018)

A co-citação é uma medida de correlacionamento (similaridade semântica) entre dois documentos (fontes ou autores). Um acoplamento bibliográfico entre esses dois documentos ocorre quando estes fazem referência simultânea a um terceiro documento (fonte ou autor). A rede de cocitação de fontes apresentada na Figura 8 evidencia os periódicos e anais de eventos mais co-citados (relacionados pelos temas) pelas referências do Portfólio. O tamanho dos círculos (representativo de cada periódico) relaciona-se ao número de citações recebidas dentro do portfólio. Nesta rede, apenas os periódicos com três ou mais citações foram representados (definido na construção da rede). A distância entre os círculos se relaciona a proximidade temática dos periódicos. O VOSviewer realiza a divisão dos periódicos por clusters temáticos, assim, foram identificados 4 CLUSTERS principais relacionados: 1. (VERDE) à GESTÃO e ao MARKETING, dentre os quais se destaca o periódico Harvard Business Review; 2. (VERMELHO) à GESTÃO e ao DESIGN, dentre os quais se destaca o periódico Journal of Service Research; 3. (AZUL) à GESTÃO, dentre os quais se destaca o periódico Journal of Operations Management; e 4. (ROSA) ao DESIGN, dentre os quais se destaca o periódico Internacional Journal of Design. 
Figura 8 - Rede de co-citação de fontes

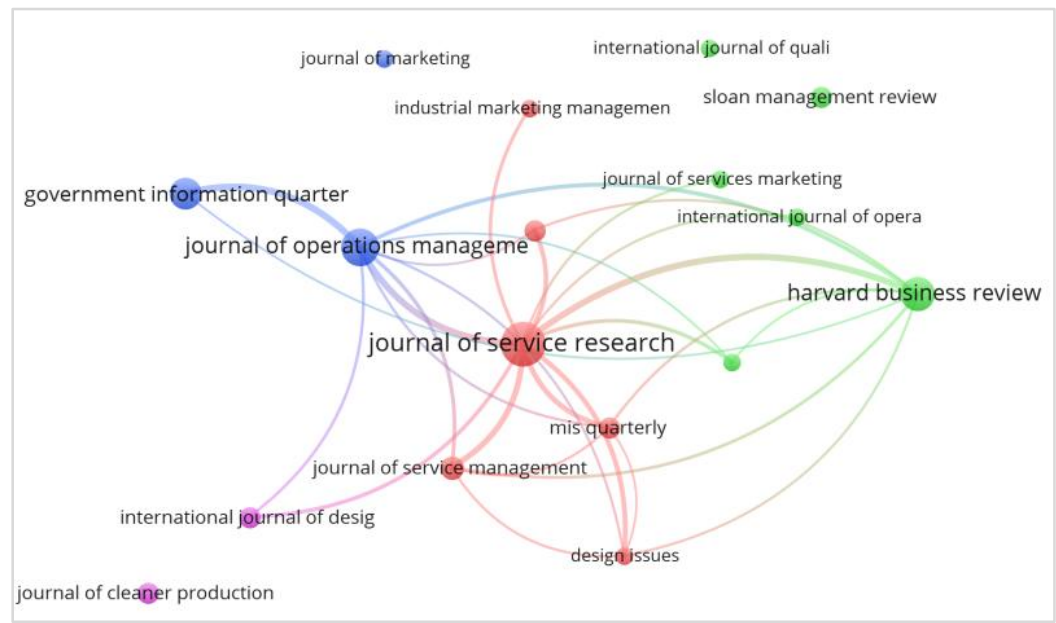

Fonte: Elaborado pelo autor com base na lista criada na Scopus (2018)

Dentre os periódicos apresentados nas Figuras 7 e 8, destacam-se o "Research Technology Management" e o "Simulation And Gaming" que publicam pesquisas na área de prototipagem de serviços e gamificação com vistas a Gestão de Design. Os demais estão voltados à área de prototipagem de serviços com vistas a Gestão de Design: Journal of Service Research; International Journal of Quality and Reliability Management; Sustainability; Technology in Society; International Journal of Quality and Services Sciences; South African Journal of Industrial Engineering; Kybernetes; Info; Journal of Operations Management Internacional Journal of Design; Journal of Service management; e Design Issues.

$\mathrm{Na}$ análise bibliométrica (Figuras 9 e 10) não foi possível identificar os principais grupos de pesquisa, pesquisadores e instituições, já que possui uma referência de cada item.

Figura 9 - Rede de Co-citação de Autores e Documentos por Autor

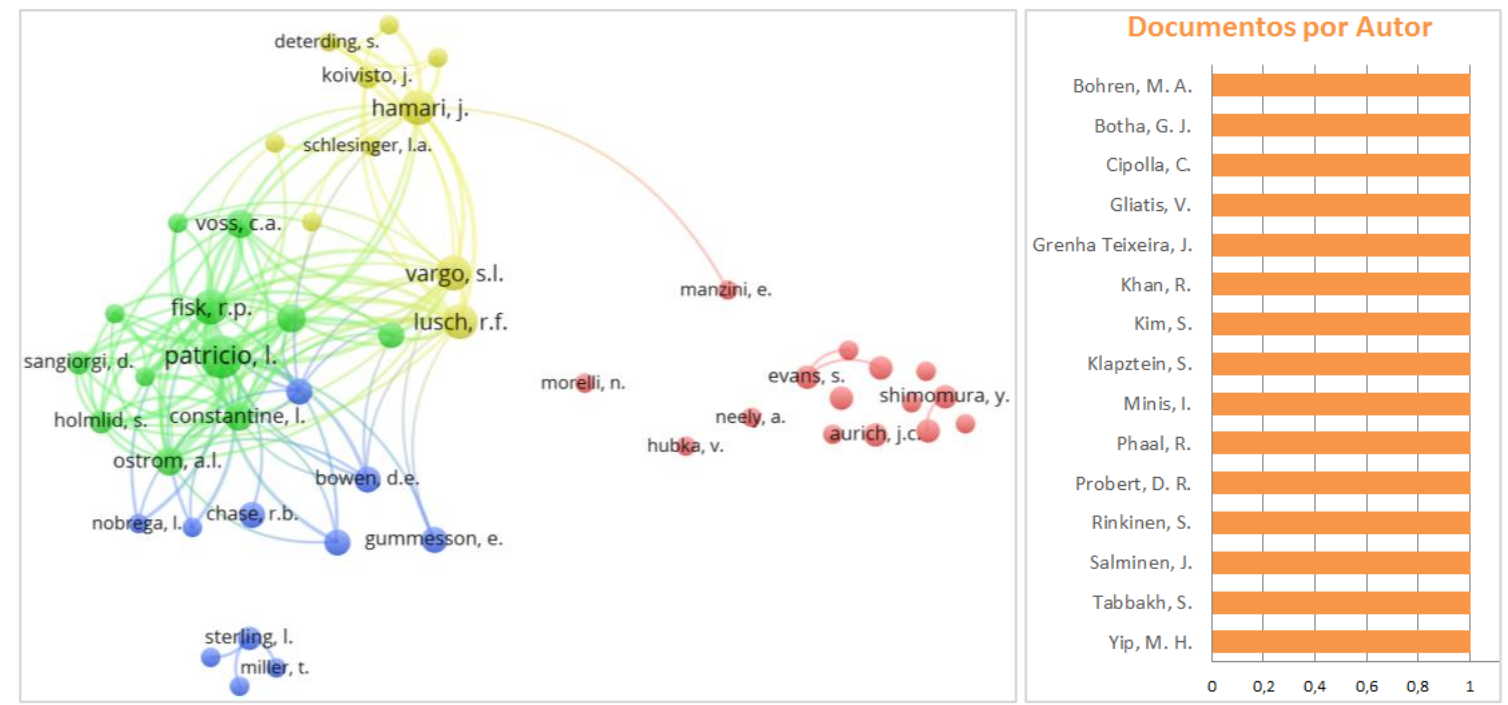

Fonte: Elaborado pelo autor com base na lista criada na Scopus (2018)

No entanto, a rede de co-citação dos artigos tornou possível detectar os principais autores. A rede de co-citação de autores apresentada na Figura 9 evidencia os principais autores mais co-citados 
pelas 13 referências do Portfólio Final. $\mathrm{O}$ tamanho dos círculos da rede (representativo de cada autor) relaciona-se ao número de citações recebidas dentro do portfólio. Nesta rede, apenas os autores com três (3) ou mais citações foram representados (definido na construção da rede). O VOSviewer realiza a divisão dos autores por clusters temáticos. A partir desta rede, identificaram-se 4 CLUSTERS de autores: 1. (VERDE) Se destaca os autores PATRICIO (13 citações) e FISK (9 citações); 2. (AMARELO) Se destaca os autores HAMARI, VARGO e LUSCH, todos com 9 citações cada; 3. (AZUL) Se destaca os autores BOWEN, GUMMESSON e CHASE, todos com 5 citações cada; 4. (VERMELHO) Se destaca os autores EVANS, SHIMOMURA e AURICH, todos com 4 citações cada; Destaca-se também os autores MANZINI, MORELI e HUBKA pela proximidade com os demais clusters, todos com 3 citações.

Figura 10 - Documentos por Instituição e por Território

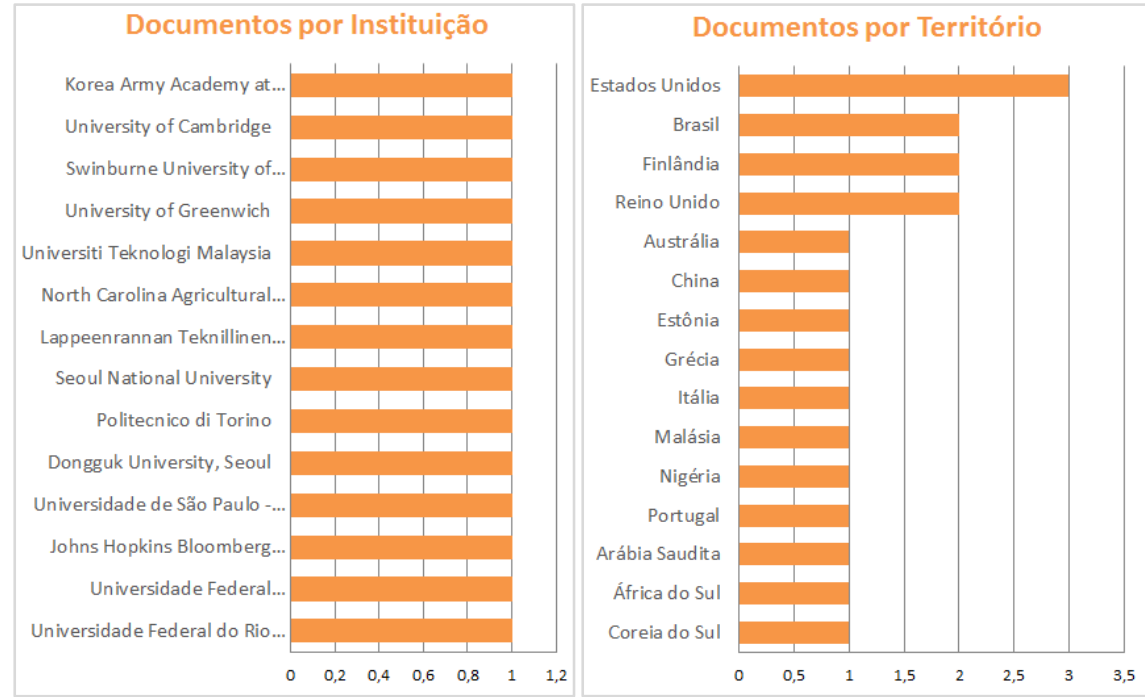

Fonte: Scopus (2018)

A análise bibliométrica (Figura 10) permitiu apontar que o país que mais publica nessa área é os Estados Unidos, seguido pelo Brasil, Finlândia e Reino Unido. Além de organizar a representação dos documentos pela área do conhecimento (Figura 11), destacando que a maioria dos artigos estão relacionados com as áreas de negócios e gestão (46\%), ciência da computação e ciências sociais (30\%), engenharia (23\%) e medicina (15\%).

Figura 11 - Documentos por área do conhecimento e Rede de Co-ocorrência de Termos

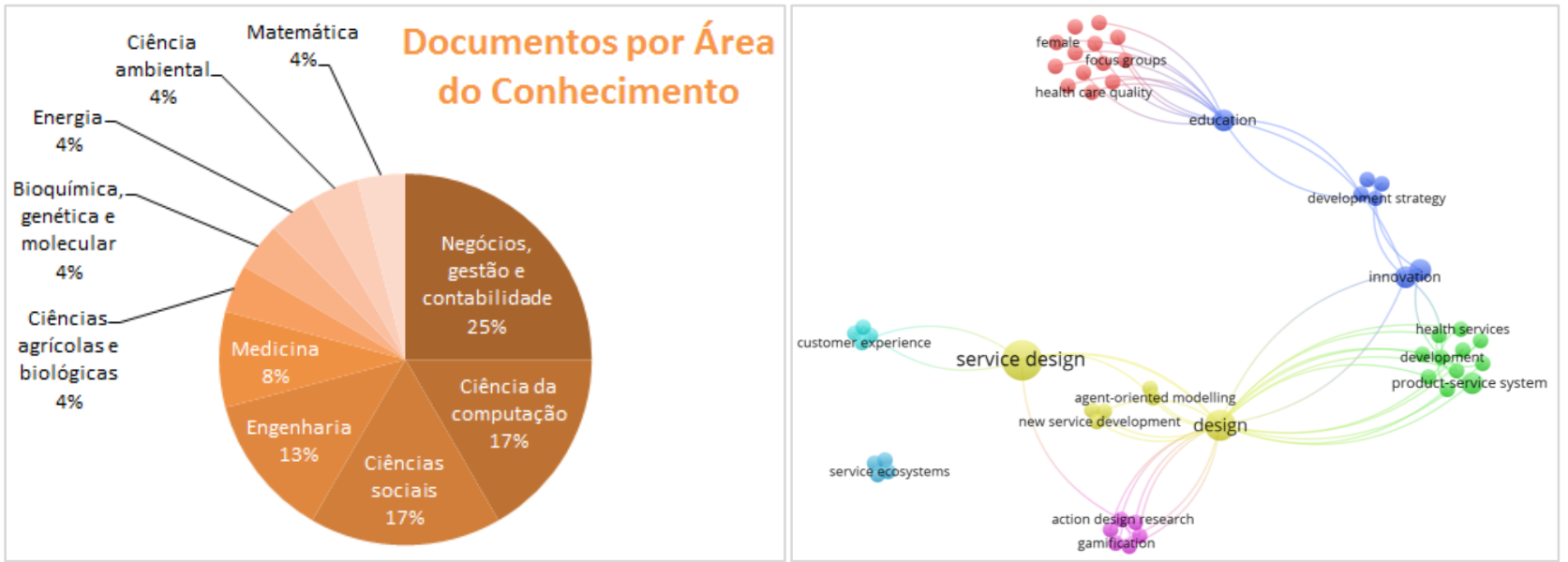

Fonte: Scopus (2018) e Elaborado pelo autor com base na lista criada na Scopus (2018) 
A rede de co-ocorrência de termos (Figuras 11 a 15) evidencia os termos mais utilizados pelas referências. O tamanho dos círculos da rede (representativo de cada termo) relaciona-se ao número de citações recebidas dentro do portfólio. Nesta rede, apenas os termos com uma ou mais citações foram representados (definido na construção da rede). A distância entre os círculos, por sua vez, se relaciona a proximidade temática dos termos. A partir desta rede, identificaram-se 7 CLUSTERS de termos. O primeiro cluster (Figura 12) apresenta os termos relacionados aos SERVIÇOS e GESTÃO da área de SAÚDE, dentre os quais se destacam os termos Health Service e Health Care Management. O segundo cluster (Figura 10) mostra os termos relacionados aos SERVIÇOS na área de GESTÃO, dentre os quais se destaca o termo Product-Service System (PSS).

Figura 12 - Clusters 1 (vermelho) e 2 (verde)

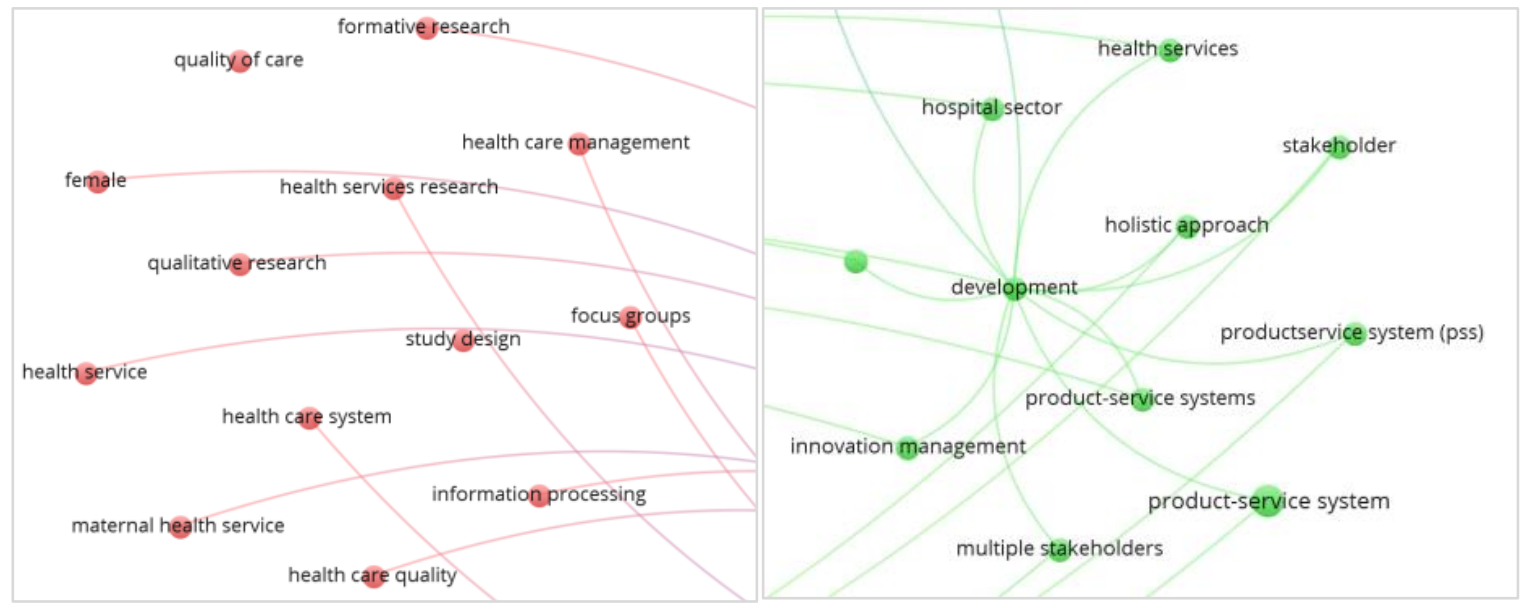

Fonte: Elaborado pelo autor com base na lista criada na Scopus (2018)

O terceiro cluster (Figura 13) exibe os termos relacionados aos SERVIÇOS nas áreas de EDUCAÇÃO e INOVAÇÃO, dentre os quais se destacam os termos Education, Innovation, Product Service System (PSS) e Service-Oriented PSS Development. O quarto cluster (Figura 14) expõe os termos relacionados ao DESIGN DE SERVIÇO, dentre os quais se destacam os termos Service Design e Design.

Figura 13 - Cluster 3 (azul forte)

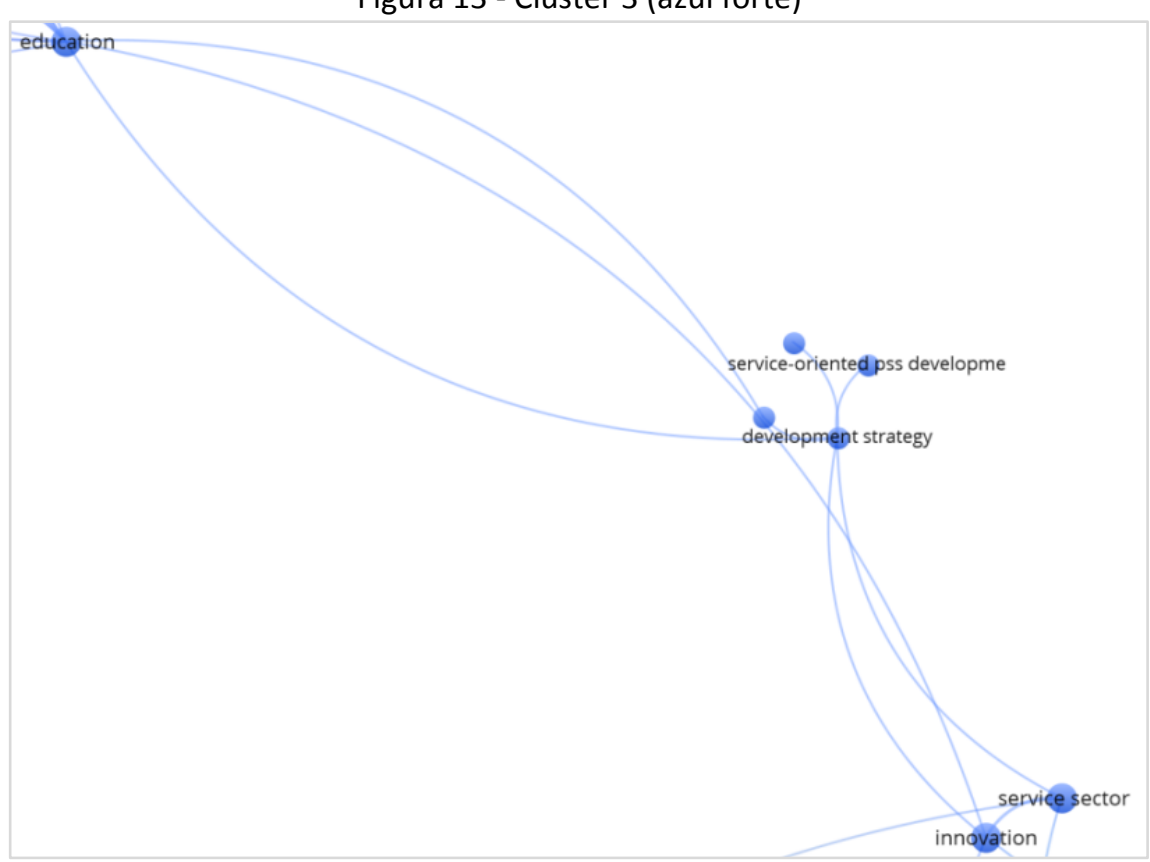

Fonte: Elaborado pelo autor com base na lista criada na Scopus (2018) 
Figura 14 - Cluster 4 (amarelo)

\section{service design}

\section{public services}

agent-oriented modelling

\section{petri nets models \\ new service development}

Fonte: Elaborado pelo autor com base na lista criada na Scopus (2018)

O quinto cluster (Figura 15) apresenta os termos relacionados aos SERVIÇOS na área de GAMIFICAÇÃO, dentre os quais se destacam os termos Gamification e Service Journey. O sexto (Figura 15) e sétimo (Figura 15) clusters evidenciam os termos relacionados ao SISTEMA DO SERVIÇO e à Sustentabilidade, dentre os quais se destacam os termos Service Systems, Service Ecosystems, Service Science, Service Recycling e Customer Experience.

Figura 15 - Cluster 5 (rosa), Cluster 6 (azul água) e Cluster 7 (azul fraco)

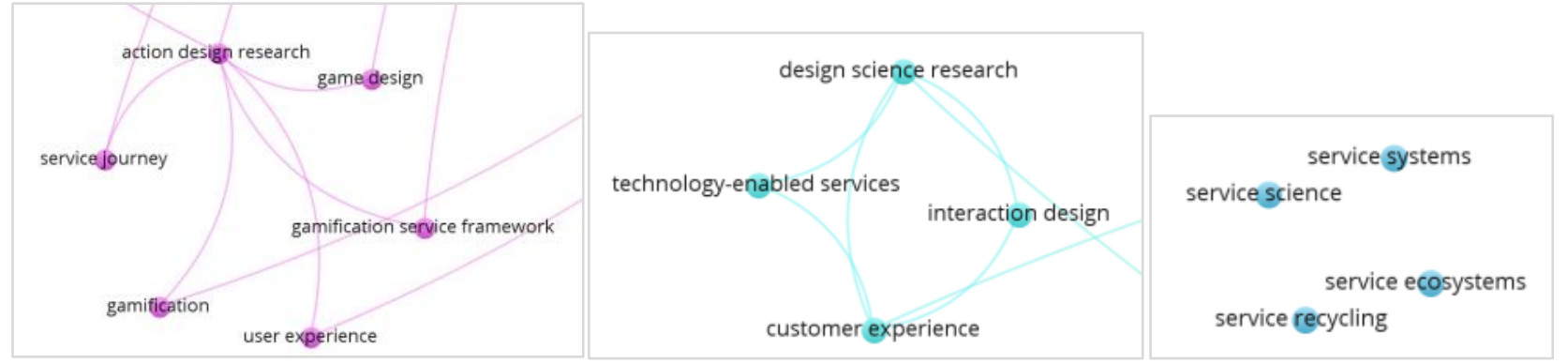

Fonte: Elaborado pelo autor com base na lista criada na Scopus (2018)

Dessa forma, evidencia-se que o Design de Serviço, e consequentemente a prototipagem de serviço, se correlacionam com as áreas de Inovação, Educação, Saúde, Marketing, Gestão, Design, Sistema, Tecnologia e Sustentabilidade. A próxima seção apresenta as considerações finais da pesquisa.

\section{Consideração Final}

Mediante o exposto nesta pesquisa, ressalta-se que o "Research Technology Management" e o "Simulation And Gaming" são os principais periódicos que publicam pesquisas na área de prototipagem de serviços e gamificação com vistas a Gestão de Design por uma abordagem sistêmica. Destaca-se, também, que a maioria dos artigos possuem abordagem teórica e empírica constituída por pesquisas primárias e secundárias. Evidencia-se, ainda, que a rede de co-citação de autores permite indicar que os principais autores, nessas áreas, são L. PATRICIO, R. P. FISK, J. HAMARI, S. L. VARGO, R. F. LUSCH. No entanto, não foi possível identificar os principais grupos de pesquisa e instituições, por causa dos resultados dispersos, porém, enfatiza-se que o país que mais publica nessa área é os Estados Unidos. Assim, conclui-se que os objetivos específicos, 
mencionados na introdução deste artigo, foram alcançados.

A RSL possibilitou identificar três pesquisas que relacionam a PS com a GD, Abordagem Sistêmica e Gamificação em que: a 1aㅡ, do autor Patrício (2016), aborda o método gamificado IdeaChef que integra conceitos de design de serviços com técnicas de resolução de problemas e inovação empresarial; a 2a ${ }^{a}$, dos pesquisadores Klapztein e Cipolla (2016), apresenta o modelo de aplicação do Gamification Service Framework que pode ser entendido como um método de prototipagem rápido para gerenciar serviços de forma simples; e a 3a , dos autores Rau, Zbiek e Jonas (2017), relata sobre o protótipo de serviço denominado "role-playing" que é usado para simular interações entre clientes e funcionários.

Diante do questionamento que induziu esta pesquisa, "A gamificação vem sendo utilizada como uma ferramenta ou método para prototipar serviços?", ressalta-se que os três artigos mencionados anteriormente utilizaram a gamificação como uma ferramenta ou método gamificado para prototipar os serviços. No entanto, devido às poucas referências na área de gamificação, não é possível afirmar que esta pode ser considerada uma ferramenta de prototipagem de serviços.

Então, conclui-se que o objetivo geral, revisar as pesquisas realizadas sobre o processo de prototipagem de serviços por meio da gamificação, com vistas à gestão de design por uma abordagem sistêmica, foi respondido nesta pesquisa. Porém, este artigo limitou-se em fazer a busca de pesquisas nas principais bases de dados Scopus, Scielo, Web of Science e ProQuest, o que resultou em poucas pesquisas relevantes. Por isso, sugere-se para futuras pesquisas: a expansão do portfólio de referências seja por meio da busca em outras bases de dados ou por meio da análise das referências bibliográficas citados em cada artigo; e a realização de uma análise qualitativa das pesquisas identificadas na RSL.

\section{Referências}

AROS, K. C.; FIGUEIREDO, L. F. G. Gestão de design para inovação social: uma possível relação. In: Fourth International Conference on Integration of Design, Engineering and Management for innovation, Florianópolis, 2015. Anais do 4ㅇIDEMI. Florianópolis: UDESC, 2015.

BERTALANFFY, L V. Teoriageral dos sistemas: fundamentos, desenvolvimentoe aplicações. 8. ed. Petrópolis, RJ: Vozes, 2014.

BLOMKVIST, J. Conceptualising Prototypes in Service Design. Tese (doutorado) - Department of Computer and Information Science, Faculty of Arts and Sciences, Linköping University, Suécia, 2011.

BOHREN, M. A. et al. Formative research and development of innovative tools for "Better Outcomes in Labour Difficulty" (BOLD): study protocol. Reproductive Health, v. 12, 2015.

BROWN, T. Design thinking: uma metodologia ponderosa para decretar o fim das velhas ideias. Rio de Janeiro: Elsevier, 2010.

BOTHA, G. J.; KRUGER, P. S.; DE VRIES, M. Enhancing customer experience through business process improvement: an application of the Enhanced Customer Experience Framework (ECEF). South African Journal of Industrial Engineering, v. 23, n. 1, p. 39-56, maio 2012.

CAPRA, F. A teia da vida: uma nova compreensão científica dos sistemas vivos. 12. ed. São Paulo: Cultrix, 2010.

CASTRO, G. V. de. Jardins comunitários de Nova York: um método para recomendações baseado no design de experiência. Tese (doutorado) - Departamento de Artes e Design, Pontifícia 
Universidade Católica do Rio de Janeiro, Rio de Janeiro, 2014.

COHEN, A. M. The gamification of education. Futurist, v. 45, n. 5, p. 16-17, 2011.

CONFORTO, E. C.; AMARAL, D.; SILVA, S. L. da. Roteiro para revisão bibliográfica sistemática: aplicação no desenvolvimento de produtos e gerenciamento de projetos. In: Congresso Brasileiro de Gestão e Desenvolvimento de Produto, 8., Porto Alegre, 12 a 14 set. 2011. Anais do 8o Congresso Brasileiro de Gestão e Desenvolvimento de Produto. Porto Alegre: UFRGS, 2011.

CUTHBERTSON, R.; FURSETH, P.I. The Service Innovation Triangle: a tool to create value through innovation. ISPIM Conference Proceedings, Manchester, p. 1-13, 2012.

DALLAGNOL, V. A inserção da gamificação no processo de gestão de design. Dissertação (mestrado) - Centro de Comunicação e Expressão, Programa de Pós-Graduação em Design, Universidade Federal de Santa Catarina, Florianópolis, 2016.

DA SILVA, J. G.; URDAN, A. T. Three integrating service management models: a comparative on methodological criteria. Revista Brasileira de Marketing, v. 15, n. 5, p. 699-711, 2016.

ELHAG, A.A. et al. A Systematic Composite Service Design Modeling Method Using Graph-Based Theory. PLoS One, San Francisco, v. 10, n. 4042015.

GIL, A. C. Como elaborar projetos de pesquisa. São Paulo: Atlas, 2010.

GLIATIS, V.; MINIS, I.; KIRIAKI, M. L. Assessing the impact of failures in service operations using experimental design with simulation. The International Journal of Quality \& Reliability Management, Bradford, v. 30, n. 1, p. 23-46, 2013.

GRENHA TEIXEIRA, J. et al. The MINDS Method: Integrating Management and Interaction Design Perspectives for Service Design. Journal of Service Research, v. 20, n. 3, 2016.

HOSS, M. J. Prototipagem de serviços: um estudo exploratório com foco na iluminação de habitações de interesse social. Dissertação (Mestrado) - Setor de Artes, Comunicação e Design, Universidade Federal do Paraná, Curitiba, 2014.

HUOTARI, K.; HAMARI, J. Defining gamification: a service marketing perspective. In: Proceeding of the 16th International Academic MindTrek Conference, ACM New York, pp. 17-22, 2012.

$\mathrm{KIM}, \mathrm{S}$. et al. Development of an Innovation Model Based on a Service-Oriented Product Service System (PSS). Sustainability, v. 7, n. 11, p. 14427-14449, 2015.

KLAPZTEIN, S.; CIPOLLA, C. From Game Design to Service Design. Simulation \& Gaming, v. 47, n. 5, p. 566-598, 2016. KOTLER, P. Administração de Marketing. São Paulo: Prentice Hall, 2005.

MARTINS, R. F. de F.; MERINO, E. A. D. A gestão de design como estratégia organizacional. 2. ed. Londrina: Eduel; Rio de Janeiro: Rio Books, 2011.

MASTROGIACOMO, L.; BARRAVECCHIA, F.; FRANCESCHINI, F. Service recycling and ecosystems: an intriguing similarity. International Journal of Quality and Service Sciences, v. 8, n. 4, p. 555-562, 2016.

MCGONIGAL, J. A realidade em jogo. Rio de Janeiro: BestSeller, 2012.

MORITZ, S. Service Design: practical access to an evolving fiel. Köln International School of Design, 2005.

MOZOTA, B. B. Gestão do design: usando o design para construir o valor de marca e inovação corporativa. Porto Alegre: Bookman, 2011.

NAVARRO, G. Gamificação: a transformação do conceito do termo jogo no contexto da pós-modernidade. 
In: CELACC, 2013. (http://200.144.182.130/celacc/sites/default/files/media/tcc/578-1589-1-PB.pdf)

OLENSKI, S. How to Solve with Gamification. In: Forbes, 2014. (http://www.forbes.com/sites/steveolenski/2014/03/05/how-to-solve-your-biggest-marketing-problems-with-gamification/)

PATRÍCIO, R. ideaChef ${ }^{\circledR}$ : A gamified approach for engaging teams in corporate innovation \& entrepreneurship. In: International Society for Professional Innovation Management Conference Proceedings, Manchester, 2016. Anais do ISPIM Conference Proceedings, 2016. Manchester: 2016.

RAU, C.; ZBIEK, A.; JONAS, J.M. Creating Competitive Advantage from Services: a Design Thinking Case Study from the Commodities Industry. Research Technology Management, Arlington, v. 60, n. 3, p. 48-56, May 2017.

SALEN, K.; ZIMMERMAN, E. Rules of play: Game design fundamentals. Cambridge, MA: The MIT Press, 2004. (https://gamifique.files.wordpress.com/2011/11/1-rules-of-play-game-design-fundamentals.pdf)

SALMINEN, J.; RINKINEN, S.; KHAN, R. Developing a regional design support service. Info: the Journal of Policy, Regulation and Strategy for Telecommunications, Information and Media, Bradford, v. 17, n. 4, p. 81-90, 2015.

SCOPUS. Analyze search results. In: Scopus, 2017. (https://www.scopus.com/search/form.uri?display=basic)

SILVA, C.S.; FIGUEIREDO, LF.Abordagem Sistêmicada Gestãode Designem Microempresase Empresas de Pequeno Porte(MPES). In:9 P\&D Design-Congresso Brasileiro dePesquisae Desenvolvimentoem Design,2010, SãoPaulo. P\&D Design 2010, 2010.

SILVA, C. S.; FIGUEIREDO, L. F. G. Abordagem Sistêmica em MPEs: design de sistemas para elaboração de ferramentas de gestão de design. In: 10 P\&D Design - Congresso Brasileiro de Pesquisa e Desenvolvimento em Design, 2012, São Luiz. P\&D Design 2012. São Luiz: Anais P\&D Design 2012, 2012.

SIRENDI, R. Designing Proactive Public Senvices as Sociotechnical Systems by Using Agent-Oriented Modelling. European Conference on e-Government. Anais de Kidmore End: Academic Conferences International Limited, jun. 2016.

STICKDORN, M.; SCHENEIDER, J. This is service design thinking: Basics-tools-Cases. In: Edmund Lik-Ching Fung, 2011. (http://www.edmundlfung.com/files/books/This\%20is\%20Service\%20Design\%20Thinking \%20-\%20Stickdorn\%20Marc,\%20Schneider\%20Jakob.pdf)

TABBAKH, S. et al. Petri Nets Application and Implementation on New Service Development. IIE Annual Conference. Proceedings, p. 1-10, 2012.

VIANNA, Y.; VIANNA, M.; MEDINA, B.; SAMARA; T. Gamification, inc: como reinventar empresas a partir de jogos. 1 ed. Rio de Janeiro: MJV Press, 2013.

VOSVIEWER. Visualizing scientific landscapes. In: Vos Viewer, 2017. (http://www.vosviewer.com/) YIP, M. H.; PHAAL, R.; PROBERT, D. R. Characterising product-service systems in the healthcare industry. Technology in Society, v. 43, p. 129-143, 2015.

$\mathrm{ZHU}, \mathrm{H}$;; GAO, J.; CAI, Q. A product-service system using requirement analysis and knowledge management technologies. Kybernetes, London, v. 44, n. 5, p. 823-842, 2015.

ZIMMERSANTOS, G. F.; HOFFMANN, M. G. Pursuing effectiveness in public administration: a proposal for a method to design and implement public senvices in the city offlorianopolis. NAVUS-Revista de Gestão e Tecnologia, v. 6, n. 1, p. 88-105, 2016. 